\title{
CHARACTERISTICS OF THE JET IMPACT DURING THE INTERACTION BETWEEN A BUBBLE AND A WALL
}

\author{
LI SHUAI \\ College of Shipbuilding Engineering, Harbin Engineering University, \#145, Nantong Street, Harbin, 150001, \\ China \\ hgcls@163.com \\ WANG SHI-PING \\ College of Shipbuilding Engineering, Harbin Engineering University, \#145, Nantong Street, Harbin, 150001, \\ China \\ wangshiping@hrbeu.edu.cn \\ ZHANG A-MAN \\ College of Shipbuilding Engineering, Harbin Engineering University, \#145, Nantong Street, Harbin, 150001, \\ China \\ zhangaman@hrbeu.edu.cn
}

Published 22 June 2016

\begin{abstract}
The dynamics of a toroidal bubble splitting near a rigid wall in an inviscid incompressible fluid is studied in this paper. The boundary integral method is adopted to simulate the bubble motion. After the jet impact, the vortex ring model is used to handle the discontinued potential of the toroidal bubble. When the toroidal bubble is splitting, topology changes are made tear the bubble apart. Then, the vortex ring model is extended to multiple vortex rings to simulate the interaction between two toroidal bubbles. A typical case is discussed in this study. Besides, the velocity fields and pressure contours surrounding the bubble are used to illustrate the numerical results. An annular high pressure region is generated at the splitting location, and the maximum pressure may be much higher than the jet impact. More splits may happen after the first split.
\end{abstract}

Keywords: Bubble dynamics; jet; split; toroidal bubble; multiple vortex rings.

\section{Introduction}

The dynamic behavior of a bubble near a wall has been studied for nearly a century. However, there still remain some mechanisms not revealed due to the extremely rapid and complicated unsteady flow phenomena. Due to the secondary Bjerknes force from a rigid boundary, a high speed liquid jet is formed during the collapse phase of a bubble ${ }^{1}$. The bubble jet impact was used to reveal the mechanism of damage to hydraulic machinery by cavitation, as well as damage to vessels by an underwater explosion bubble ${ }^{2}$.

This is an Open Access article published by World Scientific Publishing Company. It is distributed under the terms of the Creative Commons Attribution 3.0 (CC-BY) License. Further distribution of this work is permitted, provided the original work is properly cited. 
After the jet impact, a highly localized pressure is generated at the jet tip ${ }^{3-4}$. If the bubble jet is contact with the wall, the high pressure region will be located on the wall ${ }^{5}$. However, the toroidal bubble is quite unstable and the splitting phenomenon is commonly observed in experiments ${ }^{6-8}$, which is still not yet well understood. In the present study, we adopt the boundary integral method (BIM) to simulate the bubble motion near a wall, and the vortex ring model is used to handle the discontinued potential of the toroidal bubble ${ }^{2}$. After the toroidal bubble splitting, the multiple vortex rings model $^{9}$ is employed to simulate the interaction between two toroidal bubbles. Meanwhile, the pressure in the flow field is calculated by an auxiliary function ${ }^{5}$.

\section{Theory and numerical method}

The liquid surrounding the bubble is assumed inviscid and the motion irrotational. Besides, the compressibility of the flow is ignored in the present model. Thus, the flow is governed by the Laplace equation, and the velocity potential $\varphi$ at any point in the domain could be expressed as an integral equation:

$$
\lambda(\boldsymbol{r}, t) \cdot \varphi(\boldsymbol{r}, t)=\iint_{S}\left(G \cdot \frac{\partial \varphi(\boldsymbol{q}, t)}{\partial n}-\varphi(\boldsymbol{q}, t) \frac{\partial G}{\partial n}\right) d S(\boldsymbol{q})
$$

where $\lambda(\boldsymbol{r}, t)$ is the solid angle of a fixed point $\boldsymbol{r}$ with the integration variable $\boldsymbol{q}$ also situated on boundaries, $\partial / \partial n$ is the normal outward derivative from the boundary $S$. $G=1 /|\boldsymbol{r}-\boldsymbol{q}|+1 /\left|\boldsymbol{r}-\boldsymbol{q}^{\prime}\right|$ is the half-space Green function, with $\boldsymbol{q}^{\prime}$ being the reflected image of $\boldsymbol{q}$ across the wall.

The dynamic boundary condition and kinematic boundary condition on the bubble surface can be written as:

$$
\begin{gathered}
\frac{D \varphi}{D t}=\frac{|\nabla \varphi|^{2}}{2}+\frac{P_{\infty}}{\rho}-\frac{P}{\rho}-g z \\
\frac{d \boldsymbol{r}}{d t}=\nabla \varphi
\end{gathered}
$$

where $\rho$ is the density of the liquid, $P_{\infty}$ is the ambient pressure of the liquid at the inception point of the bubble, $P$ is the pressure on the bubble surface, $g$ is the gravity acceleration.

The heat transfer, mass transfer and surface tension are also ignored in this study. Therefore, the pressure inside the bubble $P_{g}$ as a function of the volume can be described as:

$$
P_{g}=P_{0}\left(V_{0} / V\right)^{\kappa}
$$

where the subscript " 0 " denotes initial quantities, $\kappa$ is the ratio of the specific heats for the gas. 
After the jet impact, the bubble is transformed from a singly-connected into a doubleconnected form. A vortex ring is introduced inside the toroidal bubble, and the strength of the vortex ring equals the difference between $\varphi$ at the north and south poles of the bubble just before impact. Then, the velocity potential is decomposed into two parts: a single-valued remnant potential $\varphi_{r}$ and the potential induced by the vortex ring $\varphi_{v}$. The velocity is also decomposed into two parts. More details about the vortex ring model can be found in Wang's work ${ }^{2}$.

After the toroidal bubble splitting, the multiple vortex rings model $^{9}$ is used to simulate the two toroidal bubble interactions. The circulations of the toroidal bubbles are denoted by $\Gamma_{1}$ and $\Gamma_{2}$, respectively. Two mirror vortex rings are placed across the wall with the circulations being the opposite numbers of $\Gamma_{1}$ and $\Gamma_{2}$, respectively. Then, the velocity and velocity potential in the flow are decomposed into five parts:

$$
\begin{aligned}
& \varphi=\varphi_{v r 1}\left(\Gamma_{1}\right)+\varphi_{v r 2}\left(\Gamma_{2}\right)+\varphi_{v r_{-} m 1}\left(-\Gamma_{1}\right)+\varphi_{v r_{-} m 2}\left(-\Gamma_{2}\right)+\varphi_{r} \\
& \boldsymbol{u}=\boldsymbol{u}_{v r 1}\left(\Gamma_{1}\right)+\boldsymbol{u}_{v r 2}\left(\Gamma_{2}\right)+\boldsymbol{u}_{v r_{-} m l}\left(-\Gamma_{1}\right)+\boldsymbol{u}_{v r_{-} m 2}\left(-\Gamma_{2}\right)+\nabla \varphi_{r}
\end{aligned}
$$

See Ref. 9 for more details about the multiple vortex rings model. Three dimensionless parameters are used to describe the initial conditions of a bubble:

- Strength parameter: $\varepsilon=P_{0} / P_{\infty}$

- Stand-off parameter: $\gamma=d / R_{m}$

- $\quad$ Buoyancy parameter: $\delta=\left(\rho g R_{m} / P_{\infty}\right)^{0.5}$

where $R_{m}$ is the maximum radius of the bubble.

\section{Results and discussion}

In Tong's experimental observations ${ }^{3}$, there are some breakup of vapor and gas in the region of the jet tip to form many small cavities when the stand-off parameter equals 1.2. His numerical simulation met the experiment well, however, their calculation of the toroidal bubbles restricted to the pre-splitting. Similar splitting phenomena were also observed by Vogel et al. ${ }^{6}$, Lauterborn et al. ${ }^{7}$, Philipp et al. ${ }^{8}$ and Zhang et al. ${ }^{9}$. In the present calculation, the initial parameters of the bubble are set as: $\varepsilon=100, \quad \gamma=1.2, \quad \delta=0, \quad \kappa=1.25$. Since the pre-toroidal bubble dynamics have been studied in many literatures, which are not discussed in this study. Some typical phenomena of the toroidal bubble (cross-section of the bubble, the pressure contours and velocity fields) are shown in Fig. 1.

As shown in Fig. 1(a), a high pressure region is generated at the jet tip soon after the jet impact, which covers only a small area. Due to the inertia of the high speed liquid jet, a protrusion appears at the jet tip, and a 'sideways' jet moves upward on the bubble surface (frame $b \sim c$ ). The bubble is drawn towards the rigid wall during its collapsing process, and a small portion of the bubble is 'stretched out' in the vertical direction (frame c). Besides, the high pressure region becomes larger but the magnitude falls. 
It is clear that the toroidal bubble would splits into two parts, as shown in frame (c). Then, topology changes are made tear the toroidal bubble apart. The multiple vortex rings model is adopted to simulate the two toroidal bubble interactions. The velocity field and pressure contour soon after the bubble splitting is shown in frame (d). An annular high pressure region is generated at the splitting location, and the maximum pressure reaches about 270 , which is much larger than the pressure caused by the main jet impact. Zhang $\& \mathrm{Li}^{9}$ demonstrated that the maximum pressure at the splitting location is associated with the velocity differences between the two sides therein just before splitting. The bubble splitting may also contribute to the shock wave emitted.

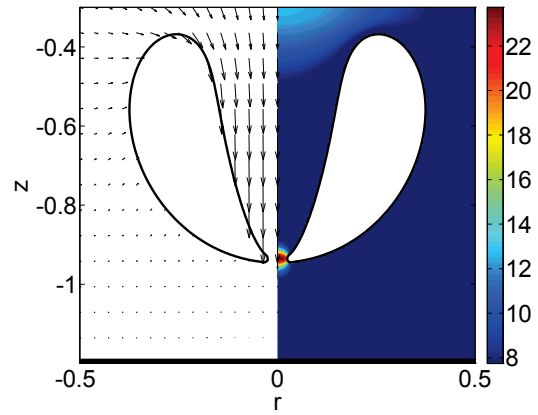

(a)

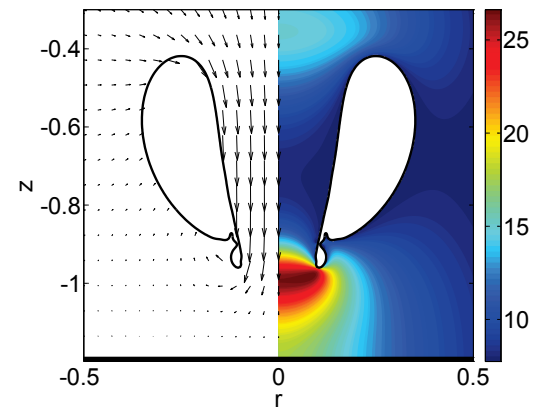

(c)

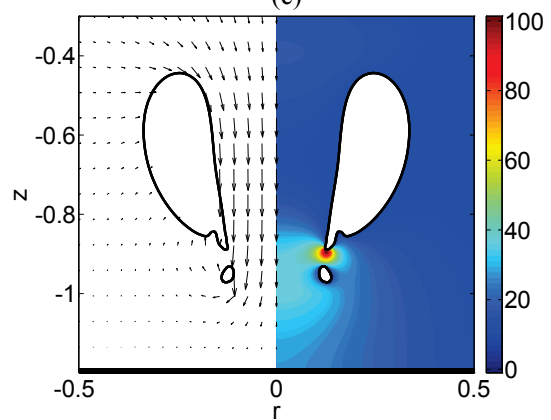

(e)

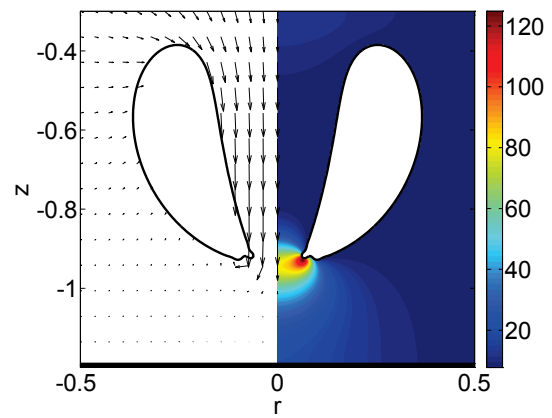

(b)

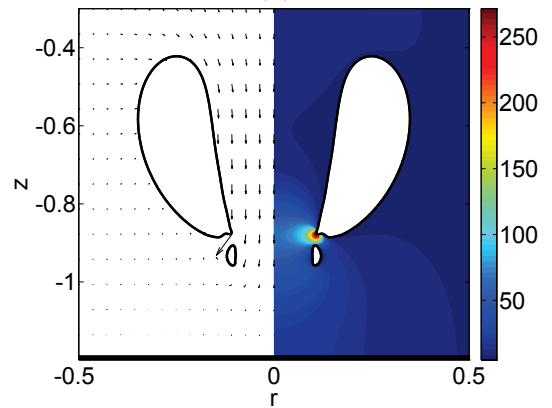

(d)

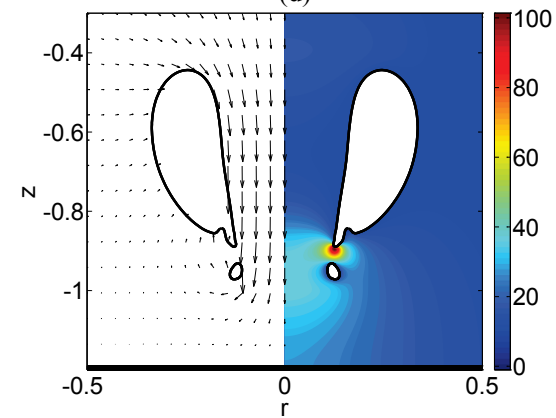

(f)

Fig. 1 Numerical simulations of a toroidal bubble motion near a rigid wall with $\varepsilon=100, \quad \gamma=1.2, \delta=0$. The location of the rigid boundary is the lower limit of the frames. The non-dimensional times of the numerical results are: $2.2657,2.271,2.282,2.2823,2.2823,2.29$ and 2.2982 , respectively. 
After the splitting, the maximum pressure at the splitting location decreases gradually. However, the high pressure region at the jet tip still exists because the main jet keeps impacting on the rigid wall due to inertia (frame $\mathrm{d} \sim \mathrm{f}$ ). Meanwhile, a protrusion appears at the bottom of the upper toroidal bubble again. As shown in frame (f), the upper toroidal bubble would split once again, which won't be discussed in the present study. The numerical simulations of this case are similar with the experimental results in Tong ${ }^{3}$ and Brujan $^{4}$.

\section{Conclusions}

In the present study, the boundary integral method is used to study the characteristics of the jet impact during the interaction between a bubble and a rigid wall. After the jet threading the opposite side of the bubble, a vortex ring is placed inside the toroidal bubble. The pressure contour shows the high pressure caused by the jet impact. The toroidal bubble splits when an annular 'sideways jet' collides with the main jet, generating an annular high pressure region at the splitting location. Our numerical results shows more splits may happen after the first split.

\section{Acknowledgments}

This work is supported by the Specialized Research Fund for the Doctoral Program of Higher Education (20122304110001), the Harbin Specialized Research Fund for Scientific and technological innovation talents, and the Project Sponsored by the

Scientific Research Foundation for the Returned Overseas Chinese Scholars, State Education Ministry.

\section{References}

1. M. S. Plesset and R. B. Chapman, J. Fluid Mech. 47(2), 283-290 (1971).

2. Q. X. Wang, K. S. Yeo, B. C. Khoo, and K. Y. Lam, Theor. Comput. Fluid Dyn. 19(5), 303317 (2005).

3. R. P. Tong, W. P. Schiffers, S. J. Shaw, J. R. Blake, and D. C. Emmony, J. Fluid Mech. 380, 339-361 (1999).

4. E. A. Brujan, G. S. Keen, A. Vogel, and J. R. Blake, Phys. Fluids 14(1), 85-103 (2002).

5. S. Li, Y. B. Li, A. M. Zhang, Appl. Ocean. Res. 50, 227-236 (2015).

6. A. Vogel, W. Lauterborn, and R. Timm, J. Fluid Mech. 206, 299-338 (1989).

7. W. Lauterborn and C. D. Ohl, Ultrason. Sonochem. 4, 65-75 (1997).

8. A. Philipp and W. Lauterborn, J. Fluid Mech. 361, 75-116 (1998).

9. A. M. Zhang, S. Li, J. Cui, Physics of Fluids, 2015 (accepted) 